\title{
Totally implantable venous access systems in paediatric practice
}

\author{
A ESSEX-CATER, ${ }^{*} \mathrm{~J}$ GILBERT,$\dagger \mathrm{T}$ ROBINSON, $\dagger$ AND J M LITTLEWOOD $\dagger$ \\ ${ }^{*}$ Regional Paediatric Oncology Unit, Seacroft Hospital, Leeds and †Regional Cystic Fibrosis Unit, St James's \\ University Hospital, Leeds
}

SUMMARY Forty seven totally implantable venous access systems have been inserted in 45 patients for the management of malignant disease $(n=29)$, haematological disorders $(n=5)$, and cystic fibrosis $(n=11)$. We report our experience with them and discuss the problems we have encountered during the past three years.

If children require repeated or prolonged venous access it is accepted that long tunnelled lines improve the patients' quality of life and reduce the risk of thrombophlebitis. ${ }^{1}$ Infection of such lines is common, however, ${ }^{2}$ and even when they are not in use certain activities must be curtailed (for example, swimming), and frequent flushing of the line is needed to prevent blockage. Totally implantable systems are now available that reduce these problems. ${ }^{3}$ We have made increasing use of these systems during the past three years, and have implanted 47 in 45 children attending the regional oncology unit $(n=34)$ and regional cystic fibrosis unit $(n=13)$.

\section{Patients and methods}

Between August 1984 and January 1988, 47 totally implantable systems (46 Port-A-Cath (Pharmacia) and one Implantofix (Braun Medical)) were inserted in 45 patients. There were 18 boys and 27 girls and age at insertion ranged from 0.7 to 20 years (mean 7.9). Twenty children were less than 5 years of age. Twenty nine children were being treated for malignant disease (25 with solid tumours and four with leukaemia). Eleven had cystic fibrosis, two had thalassaemia, two had aplastic anaemia, and one had congenital red cell aplasia.

The systems were inserted for intensive or prolonged chemotherapy, repeated courses of antibiotics, or repeated blood transfusions in patients with poor venous access. Implantable systems were used if children had previously tampered with long indwelling lines or if it was thought the family would have difficulty coping with daily aseptic flushing of a tunnelled line. They were also placed in older children who did not want the more conspicuous tunnelled line and in patients where their use was likely to be intermittent (such as those with cystic fibrosis) and where daily flushing would be inconvenient and might increase the risk of infection.

The systems have been used for blood sampling, antimicrobial and cytotoxic chemotherapy, infusion of blood products, parenteral nutrition, and continuous infusion of opiates during terminal care. Though the systems have been used primarily in hospital, three have been used at home. Two children with cystic fibrosis have received a total of 14 courses of antimicrobial treatment at home, and a patient with cancer has received infusions of opiates. Patients and relatives have been taught to manage the devices successfully at home with the support of the cystic fibrosis sister (TR), permitting more regular courses of antimicrobials to be given with the least possible interference with home and school life.

\section{INSERTION}

All except one of the catheters were implanted by one of three paediatric surgeons. The catheter was inserted into the internal jugular or subclavian vein and the tip positioned in the superior vena cava. It was then tunnelled to the site of the subcutaneous pocket in which the portal was placed. The portal was then connected to the catheter and sutured in place (fig 1). The procedure was carried out under general anaesthesia except for one case, in which the system was fully replaced (in a child with cystic fibrosis) under sedation and local anaesthesia.

At first in the patients with cancer the systems were implanted on the chest wall above the nipple; more recently most have been sited below the 


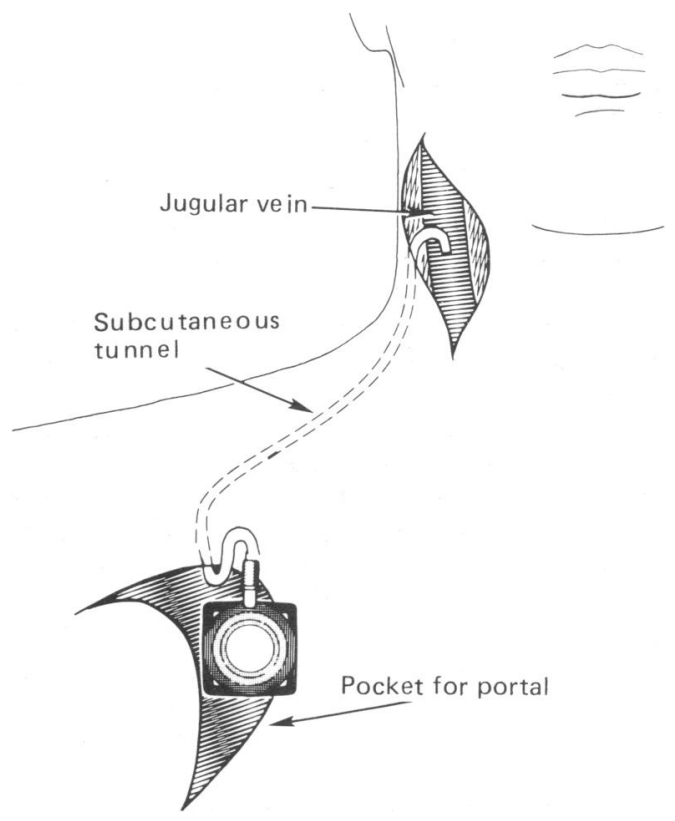

Fig 1 Insertion of Port-A-Cath.

nipple. In older girls the systems should be sited away from the breasts and the area covered by the bra, and in children with cystic fibrosis the chosen site should not interfere with chest physiotherapy. The position should be decided after discussions between the patient, parents, surgeon, and physiotherapist. In older patients who might operate their systems themselves, they should be placed in an accessible position, and away from the sensitive area round the nipple. They should be sited outside the area of any planned radiotherapy field.

\section{USE AND MAINTENANCE}

The system consists of a subcutaneous portal with a self sealing septum attached to a central venous catheter (fig 2). When in place they are totally subcutaneous and access to the portal is by a noncoring Huber needle. If an ordinary needle is used it will remove a core from the septum which will then leak. Correctly used the system will tolerate at least 1000 to 2000 perforations, depending on the size of needle used.

Insertion of the needle is done aseptically and is preceded by scrubbing an area up to three inches round the portal site for three minutes with povidone iodone solution. After palpation of the portal the needle is inserted into the septum at $90^{\circ}$ until it touches the base plate. It is important that when the needle is withdrawn the portal is held in place and positive injection pressure is maintained to prevent reflux of blood into the catheter; a closed system must be maintained at all times.

When not in use the systems must be flushed every three to four weeks using $5 \mathrm{ml}$ of heparinised saline (100 units heparin $/ \mathrm{ml})$. When in use a $90^{\circ}$ needle attached to an extension tube and three way tap should be used. The systems are flushed as the needles are removed.

Particular care must be taken to avoid mixing fluids that may cause a precipitate to be deposited in the system. After drawing blood the system should be flushed immediately with $2 \mathrm{ml}$ heparinised saline and then $20 \mathrm{ml}$ of $0.9 \%$ saline injected before the final $5 \mathrm{ml}$ of heparinised saline as the needle is removed.

In no patient has the skin overlying the portal site been denervated. In elective procedures, if the child requests it, we apply an anaesthetic cream to the portal site at least one hour before inserting the needle.

Most of the children are active during their infusions and occasionally needles are dislodged. This has not resulted in blockage of the system even if time was permitted for anaesthetic cream to be used before the needle was resited. In patients with cystic fibrosis exercise and activity are an important part of treatment and a heparin lock using a solution of 100 units $/ \mathrm{ml}$ may be used to permit freedom from the infusion during courses of treatment.

When in use for patients with cancer the skin over the portal was cleaned daily with povidone iodine solution and povidone iodine soaked gauze was placed over the entry site of the needle. This tended to dry the skin and stain the clothing, so we used

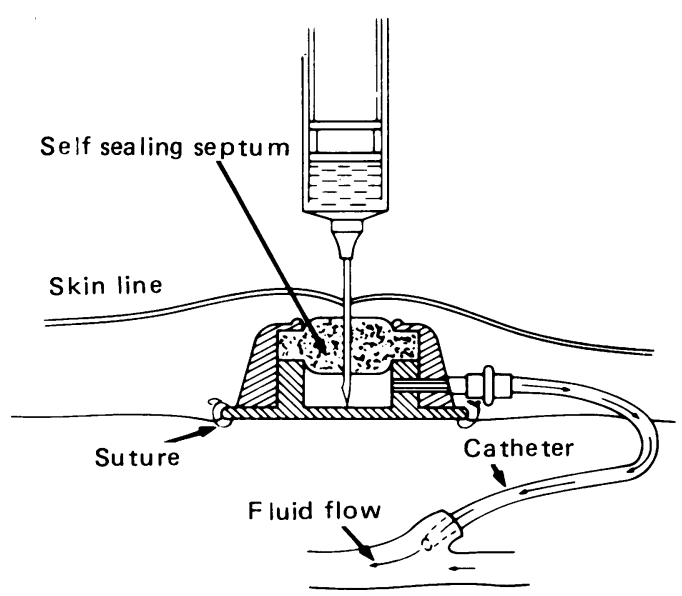

Fig 2 Access to the Port-A-Cath. 
chlorhexidine gluconate cream on the gauze rather than povidone iodine. More recently we used dry dressings that were changed each day. The needle was changed every five days.

Because patients with cystic fibrosis are not immunocompromised a different regimen of dressing and cleansing the needle site evolved. A dry dressing under an adhesive membrane was used and this was only changed when the needle was replaced (every seven days).

The systems were operated only by nurses or doctors familiar with them, or parents or older patients who were trained to use them.

Eight children were neutropenic (neutrophil concentration less than $\left.1 \cdot 0 \times 10^{9} / 1\right)$, five were thrombocytopenic (platelet concentration less than $\left.100 \times 10^{9} / 1\right)$ and one had deranged coagulation (international normalised ratio $1 \cdot 5$, kaolin cephalin clotting time 70 seconds) at the time of insertion. All thrombocytopenic patients received perioperative transfusions of platelets. The child with deranged coagulation and thrombocytopenia received intravenous vitamin $K$ and fresh frozen plasma in addition to the transfusions of platelets.

The patients with cystic fibrosis were given prophylactic antibiotics intravenously and intensive physiotherapy starting about a week before insertion of the system, and this treatment was continued afterwards until their lung function had returned to preoperative capacity. Prophylactic antibiotics were not routinely used for other patients even if they were neutropenic. Two children were already being treated with antibiotics intravenously at the time of insertion because of fever associated with neutropenia. Three patients were given short courses of antibiotics postoperative because the surgeon considered the insertion had been difficult with an increased risk of infection.

In all the patients with cystic fibrosis there was an initial decrease in respiratory function but this started to improve after 24 hours as postoperative pain decreased. In all patients with cystic fibrosis respiratory function returned to preoperative levels.

\section{Results}

The 47 systems have now been implanted for between two and 104 weeks.

The effective life of the system is taken to last until elective removal or death of the child with the system still functioning. Most of the systems are still in use, but in non-functioning systems effective life extends until the date on which the system was last known to function satisfactorily. The range of the effective life is from 0 to 104 weeks (mean 31.8). In three patients with cancer we were never able to use the system for the purpose for which it was implanted. Five systems were used to give total parenteral nutrition.

Twenty systems are no longer in use. Ten children died with the system in place. In eight it was still functioning, and in three of these patients it was used for infusion of opiates for relief of pain during terminal care. In three patients with cancer the functioning systems were removed electively under general anaesthesia after completion of courses of chemotherapy. Seven systems were removed because of complications. No child died from a problem associated with the catheter.

Patients with cystic fibrosis showed overall general improvement in respiratory function during the months after implantation, and this may be associated with the improved quality of the courses of antibiotics given intravenously - that is, doses were not missed because of difficulties in gaining venous access.

\section{COMPLICATIONS}

Seven systems have been removed because of complications, the most common of which were blockage, haemorrhage, infection, and mechanical failure.

Blockage. There have been 14 blockages in 11 children. Unblocking was attempted 11 times with either urokinase or streptokinase, and was successful in eight. In one teenager with cystic fibrosis the system became blocked three times; on the third occasion urokinase failed to clear the block, and the entire system was replaced under sedation and local anaesthesia. The second system became blocked within a week, and there was strong evidence that the patient was abusing the system.

Haemorrhage. Four children, all thrombocytopenic, had bled locally postoperatively despite prophylactic transfusions of platelets. In two the haemorrhage was severe and in one child it was impossible, even with ultrasonography, to visualise the system under the haematoma. This child had the haematoma evacuated and had no more episodes of bleeding after further transfusions of platelets and fresh frozen plasma.

The other two children each had a moderate episode of bleeding, and in one it was controlled by infusion of fresh frozen plasma and vitamin $\mathrm{K}$. The other child received no additional treatment, but her system was difficult to find because of the overlying haematoma.

Infection. Local infection of the skin occurred on six 
occasions in three patients, two with cancer and one with cystic fibrosis. Two of the patients with cancer also had systemic infections. One child had septicaemia caused by Escherichia coli at the time that a pustule appeared over the portal site; the infection resolved after treatment with ceftazidime, cloxacillin, and vancomycin given intravenously. The second child developed a skin pustule and septicaemia caused by Staphylococcus aureus that was resistant to prolonged antimicrobial treatment; the organism was grown in culture of the tip of the catheter after the system had been removed.

In a patient undergoing bone marrow transplantation the catheter blocked at the same time as Staphylococcus epidermidis was grown in cultures from both the line and the peripheral blood. No attempt was made to clear the infection and the system was removed immediately.

Two other patients developed erythema over the portal site that resolved after treatment with antibiotics given intravenously, although no organisms were grown from cultures of the blood.

One child with cystic fibrosis had three systems inserted. Her second system was removed because of the clinical suspicion of infection but this was not proved bacteriologically. She died while the third system was in place.

Mechanical problems. One child developed pain and redness over the portal site after injection of the solution of normal saline and heparin. The chest radiograph showed that the catheter tubing was lying free in the right atrium. The catheter was removed with a cardiac catheter that was inserted into the femoral vein under local anaesthesia. The rest of the system was later removed under general anaesthesia, and examination showed that the locking device was still in position over a short length of catheter that had fractured. This complication has been reported previously ${ }^{45}$ and the manufacturers have introduced a new locking device to reduce the possibility of it happening again. No patient has developed deep vein thrombosis of the arm, although this complication has been reported in adult patients. ${ }^{6}$

One patient dislodged the needle during the infusion of a solution for parenteral nutrition, some of which extravasated subcutaneously. The resulting erythema and swelling settled spontaneously.

In one patient with cystic fibrosis blood flowed back into the system during postural drainage on two occasions, but the system was flushed and the problem did not recur.

\section{Discussion}

The Port-A-Cath implantable venous access system has been a considerable advance in management in our paediatric unit. As our experience in the insertion, use, and care of these systems has increased we have encountered fewer problems. Because they are inserted in the subcutaneous tissue, the systems are comparatively tamper proof, which is useful for younger children. One child who previously had a long tunnelled line, pulled it out, and another child's tunnelled line fell out. Frequent or unexplained difficulties that occur when the systems are in use should, however, alert one to the possibility of abuse of the line.

The systems have been particularly useful in families in which social or language problems may have made daily flushing of a long tunnelled line difficult. Teenagers have expressed preference for the implantable systems; they are less conspicuous than long tunnelled lines, but should be carefully positioned as their contours may be an embarrassment. The systems do not interfere with swimming or other activities and there is no need for daily flushing. Infection of the systems has not been a particular problem, but strict asepsis in all procedures is essential.

The main benefits of implantable systems compared with long tunnelled lines are their low infection rate and the minimal care that is needed when they are not in use. The latter is a particular advantage when there are long intervals between use (such as in patients with cystic fibrosis in whom the need for good intravenous access will be lifelong but there may be intervals of several months between treatments).

Problems of bleeding should be anticipated in children with thrombocytopenia and disorders of clotting, and these should be corrected as far as possible before the operation. Insertion in patients with cystic fibrosis should be done with adequate antibiotic prophylaxis and physiotherapy if the detrimental effects of a general anaesthetic ${ }^{7}$ are to be avoided. Local anaesthetic cream is useful in those children upset by the discomfort of putting a needle into the system.

Although the initial cost of a tunnelled line is less than that of an implanted system, the higher maintenance costs of the tunnelled line make it equally expensive after four months. We are continuing to place long tunnelled lines in children in whom bone marrow transplantation is a possibility in the future.

Despite requiring a needle to gain access to the portal the totally implantable systems are well tolerated by the children, accepted by their parents, and preferred by the nursing staff. The use of these systems is now well established in the management of our patients with cancer, and their use in patients 
with cystic fibrosis who require repeated courses of antibiotics intravenously is increasing.

We thank the paediatric surgeons who inserted the devices, particularly Mr RD Spicer; the Cystic Fibrosis Research Trust for funding Dr J Gilbert (the clinical research fellow in cystic fibrosis); and the Joseph Levy Charitable Foundation for funding Sister T Robinson (the cystic fibrosis liaison sister). We also thank Dr CC Bailey and Dr IJ Lewis for sharing their experience and permitting us to report their patients.

\section{References}

${ }^{1}$ Stockwell M, Adams M, Andrew M, Cameron G, Pai K. Central venous catheters for out-patient management of malignant disorders. Arch Dis Child 1983;58:633-5.

2 Darbyshire PJ, Weightman NC, Speller DCE. Problems associ- ated with indwelling central venous catheters. Arch Dis Child 1985;60:129-34.

${ }^{3}$ McDowell HP. Hart CA. Martin J. Implantable subcutancous venous catheters. Arch Dis Child 1986;61:1037-8.

+ Martin AJ, Morris LL, Reece J, Ford WDA, Cassey J, Goldblatt E. Totally implantable vascular access in treatment of cystic fibrosis. Lancet $1986 ; \mathrm{ii}: 293$.

5 Lokich JL, Bothe A, Benotti P. Moore C. Complications and management of implanted venous access catheters.J Clin Oncol 1985:3:710-7.

6 Woll PJ, Rubens RD. Totally implantable vascular access for long term chemotherapy. Br Med J 1986;292:761.

${ }^{7}$ Price JF. The need to avoid general anaesthesia in cystic fibrosis. J R Soc Med 1986;79(suppl 12):10-2.

Correspondence to Dr J Gilbert, Regional Cystic Fibrosis Unit, St James's University Hospital, Beckett Street, Leeds LS9 7TF.

Accepted 5 August 1988 